\title{
Developing a National Registry for conditions identifiable through newborn screening
}

\author{
Jeffrey R. Botkin, MD, MPH $H^{I}$, Rebecca Anderson, $R N^{I}$, Catherine Staes, $R N, P h D^{2}$, \\ and Nicola Longo, MD, PhD
}

\begin{abstract}
Purpose: State newborn screening programs are rapidly expanding with the inclusion of a large number of uncommon conditions. There remains significant uncertainty about many aspects of these conditions including their natural history, variability, treatment modalities being used, genotype-phenotype correlations, developmental outcomes, effect on families, and costs of care, among others. Data on these important outcome variables are not collected systematically through state programs. Recently, the American Academy of Pediatrics and the federal Health Resources and Services Administration have promoted the development of a data collection system on the long-term outcomes of children with conditions identified through newborn screening. This article provides an overview of the justification for such a system and recommendations for a design. Methods: Recommendations were developed through a multidisciplinary collaboration of regional and national scholars supported through a Health Resources and Services Administration funded project. Results: We propose a registry system with data inputs from subspecialists, the Medical Home, families, and schools. Further, the proposed system would utilize emerging communication technology to provide an interactive web-based system to support families and professionals in their care of children with these complex conditions. Genet Med 2009:11(3):176-182.
\end{abstract}

Key Words: registry, newborn screening, informatics, public health

$\mathrm{N}$ ewborn dried bloodspot screening (NDBS) programs are state-based systems for early identification of children affected with heritable and congenital conditions. In recent years, programs have expanded rapidly due to the implementation of new screening technologies, primarily tandem mass spectroscopy. The state-based organization of these public health programs has led to a wide variation from state to state in many aspects of the programs. ${ }^{1}$ Although most programs provide short-term follow-up of out of range results to assure that a diagnosis has been made and treatment initiated, there have been only limited efforts to conduct long-term surveillance of children with detected disorders within state programs. A recent study by Hoff et al. ${ }^{2}$ found that $56 \%$ of NDBS programs responded that they collected no long-term follow-up (LTFU) data. More than two-thirds of state programs employed little or no use of LTFU data. When LTFU data were collected, states report using the information to track the clinical outcome of patients, track the number of individuals lost to follow-up, evaluate the performance of care providers, assess the needs of

From the ${ }^{1}$ Department of Pediatrics, Division of Medical Ethics, and ${ }^{2}$ Department of Biomedical Informatics, University of Utah, Salt Lake City, Utah.

Jeffrey R. Botkin, MD, MPH, Research Administration Building, University of Utah, 2000 East 75 South, \#108, Salt Lake City, UT 84112. E-mail: Jeffrey.botkin@hsc.utah.edu.

Disclosure: The authors declare no conflict of interest

Submitted for publication July 30, 2008.

Accepted for publication November 4, 2008.

DOI: 10.1097/GIM.0b013e318193ff0d families, and to conduct research. ${ }^{2}$ Hoff et al. ${ }^{3}$ also identified several barriers to LTFU data collection, including lack of quality assurance practices, outsourcing of program components, funding constraints, and communication challenges with care providers for affected children.

The surveys by Hoff et al.,2,3 help confirm what is common knowledge in the field, that is, most states do not systematically collect information on the longer-term outcomes of children identified through newborn screening systems. Clinics that provide long-term care to affected children are supported in many states through NDBS programs. However, evaluating the longterm outcomes of children and families identified with targeted disorders through NDBS using standardized data collection is not a significant component of state newborn screening programs in the United States.

This article outlines the need for a system for data collection on the long-term outcomes of children with conditions detectable through newborn screening. Further, we describe desirable attributes of such a system to maximize the benefits and minimize the harm of screening for children with disorders identified by NDBS, their families, care providers, and policy makers.

\section{METHODS}

These recommendations emerged from collaboration between regional and national scholars, regional and national NDBS program staff, clinicians, laboratories, public health executive leadership, and lay advocates. The project was funded by Health Resources and Services Administration (HRSA) through the Mountain States Genetics Regional Collaborative (MSRGCC) to address LTFU of children with conditions identifiable through NDBS. (Although hearing screening is an important component of state programs, our project did not formally address the potential inclusion of children and families identified with hearing disorders in a registry system.) The authors of this article were the project leaders who were responsible for the literature analysis, identification of experts, and development of consensus regarding LTFU recommendations, and convening of workgroup meetings of regional and national experts. Two workgroup meetings were held in September and December 2006. The September meeting participants included the project leaders, MSGRCC executive leadership, experts from the domains of bioethics, biomedical informatics, metabolic disorders, data system development, genetic counseling, nursing, and public health genetics, and specific representatives from the CDC Birth Defects Networks, Family Voices, HRSA Genetics Branch, NDBS follow-up programs, and both public and private NDBS laboratory programs. The product of the first meeting was a conceptual framework for the registry.

The second MSGRCC NBS Registry Meeting was convened December 14, 2006. The contributors and collaborators included additional regional and national experts as recommended, and identified by the project leaders. In addition to the participants at the September workgroup meeting, clinical he- 
moglobinopathies expertise, Logical Observation Identifiers Names and Codes (LOINCR) workgroup participant, Medical Home project expertise, National Library of Medicine Genetics Home Reference project executive leadership, National Newborn Screening and Genetic Resource Center affiliates, other regions NDBS LTFU project experts, psychosocial experts, Public Health Informatics Institute, school system expertise, state Children with Special Health Care executive leadership, state laboratory executive leadership, and statistics expertise. This Workgroup represented experts and service providers of five states of the MSGRCC and from five Regional Collaborative Centers. The draft recommendations were distributed to all meeting participants and contributors for comment although the conclusions presented in this article represent the opinions of the authors alone.

\section{RESULTS}

The provision of evidence-based care to affected children identified through NDBS and their families is challenging primarily due to the low prevalence of many of the conditions and the lack of uniform standards of care. Each individual condition targeted through NDBS programs is uncommon and many are quite rare. Hemoglobinopathies are the most common condition on NDBS panels with an incidence of $\sim 1$ in 2500 births. $^{4}$ Phenylketonuria (PKU) has an incidence of $\sim 1$ in 15,000 births. Medium chain acyl-CoA dehydrogenase deficiency and congenital adrenal hyperplasia each have an incidence of $\sim 1$ in 20,000 births. However, a number of the conditions targeted by expanded newborn screening have an incidence of $<1$ in 100,000 births including many of the disorders of amino acid and organic acid metabolism.

To understand rare, complex conditions, valid and reliable longitudinal systematic data collection with accrual of patients over a large catchment area is essential. ${ }^{5}$ At the present time, even in large referral centers, subspecialists will have only limited, if any experience with many of the conditions. The variability and wide geographic distribution associated with patient care makes randomized, controlled trials of treatment or prevention strategies difficult. It is not surprising that in a recent assessment of the evidence for treatment of inborn errors of metabolism (IEM), Steiner concluded that treatment for many conditions is not evidence-based. ${ }^{6}$ Further, there are very few metabolic specialists in the country; thus, many affected children are being followed by physicians without subspecialty training in the field. The relative lack of subspecialists may further increase the variability in treatment strategies used.

Another barrier to a reliable and valid understanding of these conditions results from ascertainment bias when the description of conditions is based on children who present clinically. Children identified secondary to the evaluation of significant symptoms are likely to represent the more severe end of the clinical spectrum for a condition. However, identification of affected individuals through population-based NBDS will likely identify many children with milder variants who may not have come to detection clinically. ${ }^{7}$ Therefore, a systematic follow-up of all children detected through newborn screening is necessary to understand the full spectrum of phenotypes associated with a condition.

The lack of long-term, systematic follow-up of affected children and their families leaves a number of significant gaps in our knowledge about these conditions and their impacts of children, families, and society. These gaps include an incomplete understanding of:

- the natural history of many of the conditions;

- the variability inherent within many conditions;
- the variability in treatment approaches;

- biochemical profiles that are associated with prognosis;

- genotype/phenotype correlations;

- demographic factors that are associated with prognosis;

- interventions that are associated with optimal outcomes;

- the benefits and harms of interventional strategies;

- costs associated with treatment;

- psychosocial impacts on families.

Further, in the absence of a clear picture of the optimal outcomes for affected children and of the interventions associated with those outcomes, quality assurance and improvement programs are not feasible. The development of a registry enables observational studies to better define optimal treatment strategies and subsequently enables monitoring of interventions to determine if optimal treatment strategies are being used. Kemper et al. ${ }^{8}$ document the discomfort many pediatricians and family practitioners feel in managing conditions included on NDBS panels. This discomfort is due to lack of familiarity with even the more common conditions on NBS panels, and the lack of standard approaches to many conditions. Standardized minimum data sets as part of a registry, perhaps in association with web-based information resources, can provide guidance to subspecialists and primary care providers to foster consistency and thoroughness during clinical encounters. Supporting quality improvement of clinical care should be a significant focus of a registry system.

The need for additional information on these rare conditions has led national organizations to call for the development of a registry for conditions identified through newborn screening. With the exception of cystic fibrosis, conditions included on NDBS panels do not have an associated population-based registry in the US. The American Academy of Pediatrics Newborn Screening Task Force ${ }^{1}$ stated that:

- the federal HRSAs Maternal and Child Health Bureau should provide grants to states to stimulate development of newborn screening information systems that are connected to the medical home, with a focus on newborn screening system process and outcome evaluation, development of standardized datasets, analyses of cost-efficiency and effectiveness, and integration with other public health data systems. Support for technological innovation (i.e., new test technologies) should include these measures.

- Pediatricians, pediatric subspecialists, and other health professionals who care for children should contribute to newborn screening data collection to advance knowledge about health outcomes and intervention effectiveness.

In 2006, HRSA's Maternal and Child Health Bureau issued a request for proposals for development of LTFU activities through the eight regional genetics collaboratives. ${ }^{9}$ The $\mathrm{Na}-$ tional Institute of Child Health and Human Development (NICHD) also is supportive of a registry as part of its development of a national translational research infrastructure for newborn screening. ${ }^{10}$ The Secretary's Advisory Committee on Heritable Disorders and Genetic Diseases in Newborn and Children is promoting LTFU that includes four components: care coordination through the medical home, evidence-based treatment, continuous quality improvement, and new knowledge discovery. ${ }^{11}$ The Secretary's Advisory Committee suggests that, ". . . registry-based approaches to discovery are needed to overcome the challenges of research in rare conditions and fill in critical gaps in the evidence base."11 To date, national organizations or advisory bodies have not developed detailed plans for a newborn screening registry. 
A registry adequate to better characterize rare conditions will need to attempt to recruit as many affected children as possible within large populations. Some individual states are sufficiently populous to have a substantial number of affected children with the more common conditions, but no state system alone is sufficient to characterize very rare conditions within a useful period of time. Therefore a registry should include multiple states within a single data collection system or be national in scope.

\section{Registries}

Registries have been created for a wide variety of purposes including product registries for those exposed to a common drug or device, health services registries for people who have had a common procedure or clinical encounter, and disease registries for those with a common diagnosis. ${ }^{12}$ In this context, a registry would consist of a database of information about children and families with conditions detected through NBDS. A registry might be relatively simple with only identifying information about the affected individual and their diagnosis. Such a registry would assist in ascertaining the prevalence of the conditions and would facilitate research by providing contact information to investigators. However, a registry also could entail a much more robust database with a variety of outcome variables collected over time for each child. A large, reliable dataset would enable a detailed description of the outcomes of affected children and the biological, environmental and therapeutic variables that are associated with those outcomes. Of course, there is a clear trade-off in registries between the feasibility of development and management on the one hand and the value of the dataset on the other. In general, it is important to limit the variables included in a registry to the minimum set necessary to address key questions to permit the maintenance of a long-term infrastructure and data quality. Defining a core minimum dataset for tracking long-term outcomes for each condition will be an essential component for building a registry system.

Registries typically do not involve research interventions per sé but can greatly facilitate access by investigators to a population of affected children and their families. As noted, Steiner ${ }^{6}$ recently reviewed the evidence base for treatment of IEM and concluded: "Treatment of IEM has historically been based to a large degree on pathophysiologic and biochemical mechanisms of disease coupled with clinical experience. A cynic might say that treatment for IEM has been based on anecdote, conjecture, theory, and tradition. Clinicians treat IEM as they were taught, with a few modifications based on clinical judgment and personal experience. As a result, evidence base is sorely needed in the field." But randomized, controlled trials of treatment strategies only become feasible when a sufficient number of children with rare conditions can be identified and recruited. ${ }^{3,13}$ Therefore, a registry is likely to be a critical component of future efforts to bring randomized, controlled trials to the management of these rare conditions.

\section{Informatics infrastructure}

Of course, the challenges posed by conditions amenable to newborn screening are not unique in health care. There are many uncommon disorders for which registries or clinical trials are underway and there is a need to synthesize information from diverse data sources spanning multiple institutions. To understand outcomes, data may be drawn directly from patients, clinicians, and parents, and indirectly from clinical, laboratory, and other computerized record systems. Although data sharing is becoming more feasible with the expanded computerization of health care records and expanded use of standards, there are still many barriers to interoperability and synthesizing information between multiple institutions. Most importantly, a single clinical entity (a laboratory test or clinical observation) may be represented in a variety of ways in different clinical information systems (i.e., using different data models, attribute names and values). As a result, data may be transferred to another system, but not understood by the receiving system. To address these and other issues, cancer researchers from the National Cancer Institute have initiated a national-scale effort, called the cancer Biomedical Informatics Grid $\left(\mathrm{caBIG}^{\mathrm{TM}}\right)$, to develop a federation of interoperable research information systems. ${ }^{14}$ caBIG will provide tools and infrastructure for institutions to map their data to a common data model and to control access to their data. Similar collaborative computer environments are under development to support large and flexible virtual organizations with multiple stakeholders in need of access to information technology resources, including physical science researchers ${ }^{15}$ and first respondents to weather-related emergencies. ${ }^{16}$ Use of caBIG tools and infrastructure, or similar cyber-infrastructure tools, may be particularly useful for automating data capture from clinical information systems to a registry for heritable disorders. Clearly, some of the information required for the registry will require hand-entry into an electronic system. However, efficient and reliable capture of a large volume of laboratory, demographic and clinical information from institutions managing multiple children with heritable disorders will require these more advanced informatics tools.

Most current registries create avenues for the unidirectional flow of information from patients and care providers to the registry's central database. The registry database then is made available to qualified investigators or quality management professionals to address relevant questions. This traditional model has been extremely valuable in many other contexts. However, contemporary information technology offers new possibilities for a bidirectional flow of information. ${ }^{17}$ That is, the registry also can be a dynamic source of information for families and care providers in their short-term and long-term management of affected children. There are emerging possibilities for developing an interactive, web-based resource for families, the Medical Home, and other care providers. The resource can include access to (1) patient-specific data (with privacy protections in place), (2) condition-specific care guidelines for acute and chronic care for use by clinicians, (3) condition-specific information for families, (4) links to trusted sources of information (e.g., the National Library of Medicine's Genetics Home Reference), and (5) listserves for sharing thoughts and experiences among interested stakeholders.

\section{Existing registries for rare heritable pediatric conditions detectable by newborn screening}

There are several existing registries that track long-term outcomes of children with heritable disorders. A review of current registries reveals five major categories of disease registries. First, there are registries developed by pharmaceutical companies that are typically focused on conditions for which their own medical products are used for treatment or research. These registries collect valuable condition-specific data for use by researchers and the pharmaceutical companies. However, many of these registries are for conditions not yet recommended for newborn screening, including Gaucher, Pompe, Fabry disease, and mucopolysaccharidosis I. ${ }^{18,19}$ Although data are collected internationally, these registries are not population-based because not every affected child is recruited for the registry. 
Second, there are state-based registries that track affected children after their diagnosis has been confirmed; however, the systems do not include systematically-collected data about long-term outcomes that can be aggregated with other statebased systems. ${ }^{2}$ In addition, there are current efforts to integrate newborn screening systems with other maternal and child health information systems, including birth defect and immunizations registries. ${ }^{20}$ Although these integrated systems will enable public health and clinical management, help avoid loss to followup, and provide prevalence information, it is unlikely that state-based registries could include enough cases or sufficient outcome data to fill the knowledge gaps noted earlier.

Third, efforts to develop regional (multistate) registries are underway. Recently, a regional condition-specific registry has been developed for MCADD. ${ }^{21}$ Developers report a 5-year expansion plan to incorporate other disorders detectable through newborn screening. This registry is designed to include LTFU data, and includes patients from multiple states. These regional registries are a valuable development and may provide sufficient cases of the more common conditions to draw valid conclusions. However, there is a risk that development of regional registries could inhibit the development of larger collaborations or a national system if regional efforts cannot be integrated due to system incompatibilities.

Fourth, nonprofit organizations have developed registries for specific disorders. For example, a national registry has been developed for cystic fibrosis. The national cystic fibrosis registry has a scope that demonstrates collaboration and use by clinicians, researchers, investors, policy makers, and families. ${ }^{22}$ The registry contains a large enough volume of patients from participating clinical centers to be useful for all of its stakeholders. This registry may provide a model for the development of a national registry for other disorders detectable through newborn screening. A similar model has been successful in Italy. ${ }^{23}$ Although there is much to learn from this model, the development of a registry by a nonprofit organization for many rare disorder is unlikely.

Fifth, national government-run registries have existed outside the United States for many years. Sweden has a unique system with a biobank containing biological samples from all children born in the country since 1975 . Blood spot samples are currently collected for PKU and four other heritable disorders. The biobank is linked to information from medical records. ${ }^{24,25}$ The integration of biological samples with medical record data provides researchers with unique opportunities for epidemiological, clinical, and evaluation studies. Participants in our project recognized the potential value of a biorepository of specimens from affected children and controls. The research value of a registry would be greatly enhanced by the development of an associated tissue repository. A biorepository would be used for research on genotype-phenotype correlations and to assess other biochemical, environmental, or physiologic associations with outcomes. Biological samples could be obtained from residual bloodspots that are retained by many state programs, ${ }^{26}$ although it would be preferable to collect specimens with informed consent after enrollment in the registry. A full consideration of the development of a regional or national biorepository of specimens relevant to NBS was beyond the scope of this project but this should be a significant focus for future discussions of a newborn screening registry.

In the United Kingdom, a PKU Registry was used to collect data about persons nationwide diagnosed with PKU following newborn screening between 1964 and 1998. ${ }^{27}$ Findings from the registry have been used to answer many important questions, including: (a) the relationship between strict, early treatment and childhood development, (b) educational progress in children with PKU, and (c) the importance of continued treatment during adult life and during pregnancy, for young women with PKU and their babies. Through these national efforts many compatibility and integration issues may be avoided and the information has been useful for clinical and research efforts over long periods of time.

All of these registries seem to meet the needs of the system developers and their current communities of users. There is a need to evaluate the strengths and limitations of these existing systems to develop a registry for conditions detectable through NDBS.

\section{Newborn screening registry design characteristics}

A regional or national registry might take many forms although there are a limited number of sources of data and outcome measures of interest. Data sources are likely to include care providers (subspecialists and primary care providers), parents or guardians, and, potentially, schools. Baseline data should include demographics and data about the diagnosis and the methods and timing of its ascertainment. Outcome data might include clinical information such as key physical examination and laboratory results, a clinical history of illnesses or complications, treatment modalities and their side effects, assessments of compliance, costs, and developmental evaluations. Relevant outcomes for families might include psychosocial assessments and financial impacts. Such a comprehensive dataset would be extremely valuable, although potentially difficult to implement, maintain, and fund.

\section{Patient population}

The total number of children potentially included in a registry is central to a system design. In the United States, there are $\sim 4$ million births each year. If the aggregate incidence of the conditions targeted is roughly 1 in $700^{28}$ then $\sim 5600$ children in the nation will be eligible for inclusion in a registry each year. Of course, state NDBS programs may choose not to collaborate with regional or national registries and families may decline to participate, so the actual number of new individuals added to the registry each year will likely be substantially smaller than 5600 . The total number of children and, potentially, adults included in a registry would continue to increase each year. The type and volume of data collected about each individual may change as the individual reaches adulthood. The system should be designed to be flexible to add conditions as they are added to newborn screening panels.

An early decision would need to be made about inclusion of a control group in the registry system. A control group of children without conditions included in NDBS panels would be necessary to formally compare affected children with unaffected children on the outcome measures under the same data collection system. Although a control group would be ideal, the complexity and costs of LTFU of unaffected children in a registry system would be great. Alternatively, outcome measures could be used that have well-validated population norms or that are derived from the National Children's Study or the National Health and Nutrition Examination Survey. Similarly, inclusion of children in the registry who are carriers of genetic conditions (heterozygotes) or children who receive initial false positive results would be valuable to better understand the outcomes of these children after testing. Again, their inclusion would be complicated and expensive. Recruitment of a group of unaffected siblings of children in the registry might offer the opportunity to evaluate a control group that would be relatively accessible and that would include both siblings who are genetic 
carriers and those who are homozygous for alleles not associated with disease. Including a small subset of children with false positive results might be feasible and worthwhile given the ongoing uncertainties about the longer-term psychosocial outcomes for these children and their families. ${ }^{1}$

\section{Recruitment}

Children and families could be recruited to the registry primarily through subspecialty clinics or the Medical Home at the completion of the diagnostic work-up of the child. Parents should be approached for permission to include the child and family in the registry with an institutional review board approved, HIPAA compliant recruitment protocol. Subsequently, data would be obtained on the child from the state health department newborn screening program. An interview of the parent to obtain demographic data could be conducted either through the medical clinic or though direct interview of the parents by registry personnel. Clinical and laboratory data from the diagnostic work-up would be input to the system.

It bears emphasis that enrollment should be conducted with the informed permission of the parents or legal guardian. Assent would be obtained from the child when he or she reaches an appropriate age. The permission process would describe fully all anticipated interventions and plans for data sharing between newborn screening programs and the regional or national registry systems. Parents also would be able to decide whether they wish to be contacted by investigators for studies that are relevant to their child. Deidentified data from the state NDBS programs would provide information on the total number of children identified in the NDBS systems so that a recruitment rate for the registry could be calculated. A recruitment rate can also be calculated using aggregate data from each state available through the National Newborn Screening \& Genetics Resource Center. ${ }^{28}$

\section{Clinical outcome measures}

Currently, there are several groups that are working to develop guidelines for clinical evaluation of children with IEM. ${ }^{19}$ (J Thomas, Personal Communication). Clinical guidelines for IEM also could be used as a model for development of similar guidelines for endocrine and hematology disorders. Such guidelines would provide recommendations on physical and developmental examinations, laboratory tests, and frequencies of evaluations. To the extent that such guidelines were used to promote a basic level of evaluation during scheduled clinical encounters, they also could be used to establish core data sets for a registry.

\section{The medical home}

Children affected with these disorders are likely to be cared by both subspecialists and primary care providers. The need for subspecialty care would depend on the severity of a particular child's condition, the availability of a subspecialist, funding constraints, and family preference. Data collection from subspecialty centers would be an essential component of the registry system and the system would need to be designed around the needs and constraints of subspecialists and their institutions. The electronic capture of data from routine clinical encounters would help minimize the burden of a registry for clinicians who see many of these children.

However, many children with mild manifestations of a condition will be seen almost exclusively by a primary care physician. Clearly, long-term outcome data will need to be collected from both types of providers. That is, subspecialty clinics should not be the sole source of data for a registry because this would bias the registry toward children with more severe manifestations. Further, some conditions, such as congenital hypothyroidism and sickle cell disease, are primarily managed through primary care providers. Unless a pediatrician or family practitioner has a particular expertise and reputation in this domain, a Medical Home involved with the registry is likely to have only one or very few affected children. Therefore, the overall burden of data entry should be modest for individual practitioners. Developing an effective mechanism for data entry in the Medical Home will be a significant challenge for a registry. Methods to motivate busy primary care offices to routinely enter accurate and complete data into the registry system would be a priority for system design.

One potential incentive for the Medical Home would be the information resources available for decision support from a registry system. Primary care providers often are uncomfortable caring for children with many of these rare and unfamiliar conditions. ${ }^{8}$ Further, the anxiety level of clinicians will be increased because some children will be susceptible to critical metabolic crises that present with common pediatric symptoms, such as vomiting. Because of the risks associated with acute deteriorations, primary care providers will be the first line of care despite their limited experience with the conditions. To support the primary care provider, information resources for the Medical Home in the registry system could include decision support guidance for acute and chronic care. Patient specific information, such as a personal health record, also may be available through the registry with access provided via a unique code held by the parents. However, child specific information will only be appropriate for clinical decision-making if the system provides accurate and timely data. Such a resource would be valuable for emergency department physicians who would be caring for the child during an acute episode but who lack ready access to up-to-date information on the child and the condition. At a minimum, the system could provide an "emergency card" that includes core information about the diagnosis, the condition, key contacts, and emergency guidance.

\section{Family impacts}

Currently, we anticipate that a registry system would work directly with parents and older children to acquire data that are not available or only partially available through care providers. This would include data on psychosocial impacts, costs of caring for affected children, developmental progress, measures of adherence to diet and/or medications, and attitude measures. A method for data entry from family interviews would require careful attention. Telephone interviews might be sufficient in many circumstances, perhaps supplemented with infrequent home visits by registry personnel, local home health, or public health professionals. Inclusion of data from family interviews or surveys would be complex and require consensus on efficient, valid instruments and sufficient staff to conduct the work and consistent efforts to promote family participation over time. Participation by families might be enhanced by creating a system that provides an element of psychological and social support. In this respect, a web-based component of the registry system might provide a platform for communication among parents and between parents and other interested parties through listserves, bulletin boards or chat rooms.

The system design should also assess the feasibility of acquiring data from assessments conducted within a child's school. School-based data include educational plans and additional psychological and functional test results for children with developmental delays or learning disabilities. Access to schoolbased data would be complex due to privacy protections in that 
domain so a careful assessment of the relative value of this information to a registry would be important.

\section{Access to the system by families and care providers}

Ideally, a registry would include a web-based tool that would be accessible to families and care providers. It may be feasible to design a system in which families would have secure access to information on their own child. Access to individual level information could be shared with care providers at the discretion of the parents. The Medical Home could have access to information and for data input on children in their care. In addition to child specific information, the system could provide access to up-to-date, professionally authored information on the relevant conditions and their care for lay readers and professionals.

\section{Access to the registry database for investigators}

Access to the registry database should be tightly controlled to protect the privacy and confidentiality of participants. Institutional Review Board approval would be anticipated for all detailed queries of the data. Individually identifiable information would not be provided to investigators without the consent of the parents. Parents should be asked during the initial consent process whether they agree to be contacted by investigators for recruitment for research; contact information for families who decline research participation would not be provided to investigators.

\section{Data collection methods}

A high priority for the selection of data collection methods will be accuracy, completeness, and low burden for clinicians and family members. Where possible, data should be transferred electronically. However, there will be a need to hand-enter selected data into the system from various sites and sources. This is not likely to be a large burden for sites that care for a small number of affected children, although any additional workload should be minimized. Referral centers that care for large numbers of affected children and their families will incur a greater burden from a registry system even if data could be captured from clinical electronic records. Funding from the registry for the additional staff time in larger centers might be necessary.

\section{System development}

Given the national scope of the registry and the breadth of stakeholders, it will be important to use a collaborative development process to gather requirements for the system. ${ }^{29}$ The key stakeholders include clinicians, public health practitioners, laboratorians, informaticians, researchers, parents, and others who are involved in the long-term management of children with heritable disorders. Their input and feedback is needed to ensure that critical components of the system are properly defined, including the specific data elements to be collected, data sources to be used, patient and provider recruitment procedures, ethical and legal requirements, quality assurance procedures, and the decision support functions, and the data access, analysis, and reporting requirements. These requirements will inform decisions that need to be made about the following informatics issues: system architecture; distributed data input to the system (including automated data collection and management of handentered data); distributed access to the system by multiple users; flexibility of system design and software tools because the relevant heritable conditions, laboratory results, and computing systems will changeover time; scalability of the system; different view of the data tailored for different stakeholders (parents, clinicians, researchers, etc); and finally, governance and administrative issues such as data hosting, security, availability, and back-up. Robust methods for tissue sample management and tracking would be necessary if a repository is associated with the registry. These issues are not insurmountable, but important to address for the success of the enterprise.

As noted earlier, a registry that is sufficiently large in scope to answer core questions in a reasonable time frame will need to be regional or national in scope. Development of such a system is challenging due to the traditional role states play in newborn screening programs. How should regional or national system be built given the existing infrastructure? Although our project was focused on the requirements for a registry system rather than the process to achieve that goal, it is likely that a "top down" development approach will be necessary. A robust, efficient registry system must be composed of subsystems that are interoperable across states and regions. Fostering unstandardized registries in each state or even in each region may result in a patchwork of systems that cannot be integrated to accomplish meaningful data sharing. In our view, it would be preferable for a national organization like the ACMG or NICHD to develop a vision for a national system and then work with states and regions to develop models and pilot components that can be extended to other states and regions in a coordinated fashion. Academic medical centers in each state and/or region should be key components as well, serving a role as leadership organizations for clinical expertise, research development, and data management.

\section{Funding}

A central challenge to the development of a registry system is funding. Support for such a system would need to be substantial and stable over a time period sufficient to achieve the basic goals. As noted, some registries are supported by pharmaceutical or device manufacturers when the purpose of the registry is to follow individuals who have received a specific intervention. Commercial funding would seem to have no significant role for a regional or national NDBS registry as we have described. Given the need for multistate collaboration for these rare conditions, the logical funding source would be the federal government. The Newborn Screening Saves Lives Act of 2007 (S 1858) was signed into law in April, 2008. Among other goals, the legislation authorizes development of a system to ". . . establish, maintain, and operate a system to assess and coordinate treatment relating to congenital, genetic, and metabolic disorders." 30 A national organization such as the NICHD or the ACMG could serve as a coordinating body for the development and maintenance of a registry, perhaps in association with the emerging translational research infrastructure.

\section{CONCLUSIONS}

Newborn screening is an important activity for the health and welfare of children. However, current state-based systems and the care of affected children are impaired by the lack of systematic LTFU. LTFU is essential to better understand the rare conditions targeted, to evaluate the current efficacy of treatment modalities, to identify strengths and weaknesses of interventions, and to establish a foundation for clinical research. The rapid and continued expansion of screening programs is not appropriate without a parallel investment in LTFU systems to assure the efficacy of these important programs. 


\section{ACKNOWLEDGMENTS}

This project was funded by HRSA through the Mountain States Genetics Regional Collaborative. Partial support for this work was provided for one author (C.J.S.) by the National Library of Medicine (Training grant \#LM007124).

We thank, Joyce Hooker, Camille Miller, and the Mountain States Regional Genetics Collaborative for their support in the conduct of this work (Subcontract \#10002615). We also thank Joanna Fanos, Susan Waisbren, Susan Berry, Linda Carr-Lee, Judy Tuerck, Janet Thomas, Chuck Norlin, Marzia Pasquali, Marie Mann, Reid Holbrook, and John Johnson for their support of the project.

\section{REFERENCES}

1. American Academy of Pediatrics. Serving the family from birth to the medical home. Newborn screening: a blueprint for the future - a call for a national agenda on state newborn screening programs. Pediatrics 2000;106: $389-422$.

2. Hoff T, Ayoob M, Therrell BL. Long-term follow-up data collection and use in state newborn screening programs. Arch Pediatr Adolesc Med 2007;161: 994-1000.

3. Hoff T, Hoyt A, Therrell B, Ayoob M. Exploring barriers to long-term follow-up in newborn screening programs. Genet Med 2006;8:563-570

4. Therrell BL, Hannon WH. National evaluation of US newborn screening system components. Mental Retard Dev Disabil Res Rev 2006;12: $236-245$.

5. Botkin JR. Research for newborn screening: developing a national framework. Pediatrics 2005;116:862-871.

6. Steiner RD. Evidence based medicine in inborn errors of metabolism: is there any and how to find it. Am J Med Genet 2005;134:192-199.

7. Wilcken B, Wiley V, Hammon J, Carpenter K. Screening newborns for inborn errors of metabolism by tandem mass spectrometry. $N$ Engl J Med 2003;348:2304-2312.

8. Kemper AR, Uren RL, Moseley KL, Clark SJ. Primary care physicians' attitudes regarding follow-up care for children with positive newborn screening results. Pediatrics 2006;118:1836-1841.

9. U.S. Department of Health and Human Services, Health Resource Service Administration Maternal Child Health Bureau, Genetics Services Branch, Division of Services for Children with Special Health Care Needs. Regional genetic and newborn screening services, HRSA-07-016, CDFA \#93.110. Program Guidance, Fiscal Year 2007

10. Alexander D, Hanson JW. NICHD research initiative in newborn screening. Mental Retard Dev Disabil Res Rev 2006;12:301-304.

11. Kemper AR, Boyle CA, Aceves J, et al. Long-term follow-up after diagnosis resulting from newborn screening: statement of the Secretary's Advisory Committee on Heritable Disorders and Genetic Diseases in Newborn and Children. Genet Med 2008;10:259-261.

12. Agency for Healthcare Research and Quality. Registries for evaluating patient outcomes: A user's guide. Rockville, MD: Prepared by Outcome DEcIDE Center [Outcome Sciences, Inc. dba Outcome] under Contract No. HHSA29020050035ITO1; 2007.

13. Alexander D, van Dyck PC. A vision of the future of newborn screening. Pediatrics 2006;117(5 Part 2):S350-S354.

14. Saltz J, Oster S, Hastings S, et al. Cagrid: design and implementation of the core architecture of the cancer biomedical informatics grid. Bioinformatics 2006;22:1910-1916

15. Truong TN, Nayak M, Huynh HH, et al. Computational science and engineering online (cse-online): a cyber-infrastructure for scientific computing. $J$ Chem Inf Model 2006;46:971-984.

16. Plale B, Gannon D, Brotzge J, et al. CASA and LEAD: adaptive cyberinfrastructure for real-time multiscale weather forecasting. Computer 2006: $56-64$.

17. Mann MY, Lloyd-Puryear M, Linzer D. Enhancing communication in the 21st century. Pediatrics 2006;117:S315-S319.

18. Disease Registries and Research.[Internet]. Cambridge: Genzyme Corporation; (C) 2008. Available at: http://www.genzyme.com/home/global_sites. asp. Accessed May 6, 2007.

19. Watson MS, Lloyd-Puryear MA, Mann MY, Rinaldo P, Howell RR. Newborn screening: toward a uniform screening panel and system-main report. Genet Med 2006;8(5 Suppl):12s-252S.

20. All Kids Count. Integration of Newborn Screening and Genetic Service Systems with Other Maternal \& Child Health Systems: A Sourcebook for Planning and Development [internet]. Decatur: Public Health Informatics Institute; 2003 June 100p. Available at: http://www.phii.org/resources/doc details.asp?id=113. Accessed May 9, 2008.

21. Region 4 Genetics Collaborative. Region 4 five year plan [Internet]. Available at: http://region4genetics.org/region4/region4_five_year_plan.aspx. Accessed July 19, 2008.

22. Cystic Fibrosis Foundation. About the cystic fibrosis foundation. Available at: http://www.cff.org/aboutCFFoundation/. Accessed November 21, 2007.

23. Registro Italiano della Fibrosi Cistica [internet]. Milan: Lega Italiana Fibrosi Cistica ONLUS; (C) 2007. Available at: http://www.fibrosicistica.it/page.php? cPath=0_40. Cited May 7, 2008.

24. Hansson SO, Bjorkman B. Bioethics in Sweden. Cambridge quarterly of healthcare ethics, Vol. 15. Cambridge: Cambridge University Press, 2006: 285-293.

25. Available at: http://www.biobanks.se/swedish.htm. Accessed July 22, 2008.

26. Olney RS, Moore CA, Ojodu JA, Lindegren ML, Hannon WH. Storage and use of residual dired blood spots from state newborn screening programs. J Pediatr 2006;148:618-622.

27. Registers [Internet]. London: UK Newborn Screening Programme Centre. 2007. Available at: http://www.ich.ucl.ac.uk/newborn/workstreams/ registers_faqs.htm. Accessed July 22, 2008

28. National Newborn Screening \& Genetic Resource Center; National newborn screening information system. Available at: http://www2.uthscsa.edu/nnsis/. Accessed November 25, 2006.

29. Public Health Informatics Institute. Taking care of business. Decatur, GA: Public Health Informatics Institute; 2006.

30. Available at: S 1858 at: http://thomas.loc.gov/cgi-bin/bdquery/z?d110: SN01858:@@@\&summ2=m\&>.Accessed July 17,2008. 